\title{
Stability and Asymptotic Behavior of a Regime-Switching SIRS Model with Beddington-DeAngelis Incidence Rate
}

\author{
Shan Wang $\mathbb{D},{ }^{1}$ Youhua Peng $\mathbb{D},{ }^{1}$ and Feng Wang $\mathbb{D}^{1,2}$ \\ ${ }^{1}$ Department of Mathematics, Pingxiang University, Pingxiang 337000, China \\ ${ }^{2}$ School of Mathematics and Statistics, Central South University, Changsha 410083, China \\ Correspondence should be addressed to Youhua Peng; pengyouh@126.com
}

Received 23 March 2020; Accepted 16 June 2020; Published 15 July 2020

Academic Editor: Jürgen Pannek

Copyright (c) 2020 Shan Wang et al. This is an open access article distributed under the Creative Commons Attribution License, which permits unrestricted use, distribution, and reproduction in any medium, provided the original work is properly cited.

A regime-switching SIRS model with Beddington-DeAngelis incidence rate is studied in this paper. First of all, the property that the model we discuss has a unique positive solution is proved and the invariant set is presented. Secondly, by constructing appropriate Lyapunov functionals, global stochastic asymptotic stability of the model under certain conditions is proved. Then, we leave for studying the asymptotic behavior of the model by presenting threshold values and some other conditions for determining disease extinction and persistence. The results show that stochastic noise can inhibit the disease and the behavior will have different phenomena owing to the role of regime-switching. Finally, some examples are given and numerical simulations are presented to confirm our conclusions.

\section{Introduction}

Infectious diseases are one of the greatest enemies of human beings. Whenever they happen, they will bring great disasters to human beings. Therefore, it is of great significance to model and study the infectious mechanism of infectious diseases for disease control. SIR model, using $S(t), I(t)$, and $R(t)$ to express fractions of the susceptible, infected, and removed at time $t$, as one of the classical infectious disease models, has been studied and extended by many scholars.

Owing to the richness and importance of the research content of epidemic models, different scholars study them from different perspectives. Some authors used Lyapunov functions to study the stability of the model [1-3]. The authors in [2] proposed a new technique to study stability of an SIR model with a nonlinear incidence rate by establishing a transformation of variable. More about the stability of stochastic differential equations, we refer to $[4,5]$. Some scholars have studied the dynamic behavior of epidemic models and gave the threshold values of disease extinction and persistence so as to give control strategies for disease [6-10]. The authors in [6] have proved that the number $R_{0}^{S}$ can govern the dynamics of the model under intervention strategies by using the Markov semigroup theory. In addition, scholars have studied the ergodicity and stationary distribution of the model by making use of different methods in [11-14]. The authors in [12] generalized the method for analyzing ergodic property of epidemic models, all of which further enrich and improve the theory and application of epidemiology. Markov semigroup approach was used in [14] to obtain the existence of stationary distribution density of the stochastic plant disease system.

Parameters involved in models are more or less disturbed by some environmental noise. Mao et al. [15] have proved that the presence of noise can suppress potential population explosion, which shows that environmental noise has a great influence on the behavior of the model. In order to describe this perturbation, stochastic noise driven by continuous Brownian motion is widely studied in epidemic models and other systems with various incidence functions $[7-9,12,13,16,17]$. There are several kinds of stochastic noise, one of which is assumed that some parameters in the model are disturbed, such as the contact rate or death rate.

Beddington-DeAngelis function is an important incidence rate with the form $f(S, I)=\left(S I / m_{1}+m_{2} S+m_{3} I\right)$, 
which has been studied by some scholars [17-19]. It can be considered as a generalization of many incidence functions, for example,

(1) $m_{1}=1, m_{2}=0, m_{3}=0, f(S, I)=S I$ [7]

(2) $m_{1}=1, m_{2}=a, m_{3}=0, f(S, I)=(S I / 1+a S)[20]$
(3) $m_{1}=1, m_{2}=0, m_{3}=a, f(S, I)=(S I / 1+a I)[13]$

(4) $m_{1}=0, m_{2}=1, m_{3}=1, f(S, I)=(S I / S+I)[21]$

Hence, a certain SIRS model containing constant population size and stochastic perturbation takes the following form:

$$
\left\{\begin{array}{l}
\dot{S}(t)=\left(\mu-\mu S(t)-\frac{\beta S(t) I(t)}{m_{1}+m_{2} S(t)+m_{3} I(t)}+\theta R(t)\right) \mathrm{d} t-\frac{\sigma S(t) I(t)}{m_{1}+m_{2} S(t)+m_{3} I(t)} \mathrm{d} B(t), \\
\dot{I}(t)=\left(\frac{\beta S(t) I(t)}{m_{1}+m_{2} S(t)+m_{3} I(t)}-[\mu+\gamma+d] I(t)\right) \mathrm{d} t+\frac{\sigma S(t) I(t)}{m_{1}+m_{2} S(t)+m_{3} I(t)} \mathrm{d} B(t), \\
\dot{R}(t)=(\gamma I(t)-[\mu+\theta] R(t)) \mathrm{d} t,
\end{array}\right.
$$

where $\mu$ represents the birth and death rate, $\beta$ denotes the valid contact coefficient, $\gamma$ means the rate at which the infected is cured and returns to the removed, $d$ is the death rate due to disease, $\theta$ expresses the rate of losing immunity and returning to the susceptible, $\sigma$ represents the intensity of stochastic perturbation, and $B(t)$ is the standard Brownian motion.

Moreover, the environment in our life often changes, for example, the seasons, temperature and humidity will always change and the mechanism and infectious ability of diseases will change accordingly. Therefore, the parameters in the model will change suddenly and discontinuously, which cannot be depicted by continuous Brownian motion, but can be described by continuous-time Markov chain in finite- state space. Many scholars have studied the epidemic models with Markovian regime-switching, see $[8-10,12]$. Due to the rationality and significance of multiple environments in the model, regime-switching is also applied in population model and other fields, see $[18,22,23]$. We refer the readers to $[5,24,25]$ for the theory and more knowledge of Markovian switching.

As far as we know, although there are a great many number of research studies on the epidemic models with Markovian switching, there is little work on the properties of the regime-switching SIRS model the with Beddington-DeAngelis incidence rate. In this paper, we will discuss the properties of this kind, and its expression is as follows:

$$
\left\{\begin{array}{l}
\dot{S}(t)=\left(\mu\left(\alpha_{t}\right)-\mu\left(\alpha_{t}\right) S(t)-\frac{\beta\left(\alpha_{t}\right) S(t) I(t)}{m_{1}\left(\alpha_{t}\right)+m_{2}\left(\alpha_{t}\right) S(t)+m_{3}\left(\alpha_{t}\right) I(t)}+\theta\left(\alpha_{t}\right) R(t)\right) \mathrm{d} t \\
-\frac{\sigma\left(\alpha_{t}\right) S(t) I(t)}{m_{1}\left(\alpha_{t}\right)+m_{2}\left(\alpha_{t}\right) S(t)+m_{3}\left(\alpha_{t}\right) I(t)} \mathrm{d} B(t), \\
\dot{I}(t)=\left(\frac{\beta\left(\alpha_{t}\right) S(t) I(t)}{m_{1}\left(\alpha_{t}\right)+m_{2}\left(\alpha_{t}\right) S(t)+m_{3}\left(\alpha_{t}\right) I(t)}-\left[\mu\left(\alpha_{t}\right)+\gamma\left(\alpha_{t}\right)+d\left(\alpha_{t}\right)\right] I(t)\right) \mathrm{d} t \\
+\frac{\sigma\left(\alpha_{t}\right) S(t) I(t)}{m_{1}\left(\alpha_{t}\right)+m_{2}\left(\alpha_{t}\right) S(t)+m_{3}\left(\alpha_{t}\right) I(t)} \mathrm{d} B(t), \\
\dot{R}(t)=\left(\gamma\left(\alpha_{t}\right) I(t)-\left[\mu\left(\alpha_{t}\right)+\theta\left(\alpha_{t}\right)\right] R(t)\right) \mathrm{d} t,
\end{array}\right.
$$

where $\alpha_{t}$ is a continuous-time finite-state Markov chain taking values in space $\mathrm{E}=\{1,2, \ldots, N\}$ with transition rate

$$
\mathbb{P}\left(\left|\alpha_{t+\Delta t}=j\right| \alpha_{t}=i\right)= \begin{cases}q_{i j} \Delta t+o(\Delta t), & \text { if } i \neq j, \\ 1+q_{i i} \Delta t+o(\Delta t), & \text { if } i=j,\end{cases}
$$


for a sufficiently small $\Delta t>0$. We assume in this paper that the matrix is conservative and irreducible, which signifies that the unique stationary distribution $\pi=\left(\pi_{i}\right)$ for Markov chain exists and satisfies the equations:

$$
\begin{aligned}
\pi Q & =0, \\
\sum_{i=1}^{N} \pi_{i} & =1 .
\end{aligned}
$$

The outline of this paper is organized as follows. Section 2 proves that the model has a unique positive solution and the invariant set is presented. Meanwhile, some important lemmas which will be used later are given. In Section 3, conditions of stochastic asymptotic stability in the large are established by constructing suitable Lyapunov functionals with regime-switching. In Section 4, conditions for disease extinction are discussed and condition of persistence in the mean is also studied by applying some useful inequality techniques. Section 5 presents some examples and their simulations to confirm our theoretical results.

\section{Preliminaries}

In this section, some background knowledge about differential equations with Markovian switching and several important Lemmas will be proposed, all of which will be used later in the paper.

Let $\mathbb{R}_{+}^{3}:=\left\{\left|\left(x_{1}, x_{2}, x_{3}\right)\right| x_{i}>0, \quad i=1,2,3\right\}$. We define $\left(\Omega, \mathscr{F},\{\mathscr{F}\}_{t \geq 0}, \mathbb{P}\right)$ as a complete probability space with a filtration $\{\mathscr{F}\}_{t \geq 0}$, which satisfies the usual conditions. Consider the SDEs with Markovian switching as follows:

$$
\mathrm{d} X(t)=f\left(X, \alpha_{t}\right) \mathrm{d} t+g\left(X, \alpha_{t}\right) \mathrm{d} B(t),
$$

where $f: \mathbb{R}^{n} \times \mathscr{E} \longrightarrow \mathbb{R}^{n}, g: \mathbb{R}^{n} \times \mathscr{E} \longrightarrow \mathbb{R}^{n \times m}$, and $B(t)=$ $\left(B_{t}^{1}, \ldots, B_{t}^{m}\right)^{T}$ is $m$-dimensional standard Brownian motion. For $i \in \mathscr{E}$ and any function $V(X, i) \in \mathscr{C}^{2}\left(\mathbb{R}^{n} \times \mathrm{E} ; \mathbb{R}^{n}\right)$, define the operator $\mathscr{L}$ by

$$
\begin{aligned}
\mathscr{L} V(X, i)= & V_{x}(X, i) f(X, i)+\frac{1}{2} \operatorname{tr}\left[g^{T}(X, t) V_{x x} g(X, t)\right] \\
& +\sum_{j=1}^{N} q_{i j} V(X, j) .
\end{aligned}
$$

For a differential system, people are concerned with the existence, uniqueness, form of solutions, and so on. In this paper, we are concerned about whether the model has a unique solution. Can we estimate the range of the solution more precisely? The following lemma is presented to answer these questions.

Lemma 1. For the initial value $\left(S(0), I(0), R(0), \alpha_{0}\right) \in$ $\mathbb{R}_{+}^{3} \times \mathrm{E}$, there exists a unique positive solution $\left(S(t), I(t), R(t), \alpha_{t}\right)$ for model (2). In addition, let $\Delta:=\{S, I, R>0 \mid 0 \leq N(t):=S(t)+I(t)+R(t) \leq 1\}$, then $\Delta$ is an invariant set of model (2), i.e., if $(S(0), I(0), R(0)) \in \Delta$, then $(S(t), I(t), R(t)) \in \Delta$ for all $t>0$. Moreover, $N(t) \leq \max \{N(0), 1\}$.
Proof. We take a piecemeal approach to prove. Let $0=\tau_{0}<\tau_{1}<\cdots<\tau_{n}<\cdots$ be all jump times of Markov chain $\left\{\alpha_{t}\right\}$. When $t \in\left[0, \tau_{1}\right)$, let $\alpha_{t}=c_{0}$, and we can prove that the model has a positive solution almost surely by constructing appropriate Lyapunov function. This process is common, and we omit here. When jumping to another state $\alpha_{t}=c_{1}$ for time $t \in\left[\tau_{1}, \tau_{2}\right)$, the corresponding parameters in the model will change to another set of numbers and the positive solution can be testified by the same method. Repeat this process on the intervals $\left[\tau_{2}, \tau_{3}\right),\left[\tau_{3}, \tau_{4}\right) \cdots$, and the positive solution can be obtained for $t>0$.

Then, we prove the range of the solution. Adding three equations in model (2), one has

$$
\begin{aligned}
\mathrm{d} N(t) & =\left(\mu\left(\alpha_{t}\right)-\mu\left(\alpha_{t}\right) N(t)-d\left(\alpha_{t}\right) I(t)\right) \mathrm{d} t \\
& \leq\left(\mu\left(\alpha_{t}\right)-\mu\left(\alpha_{t}\right) N(t)\right) \mathrm{d} t .
\end{aligned}
$$

For each state $i \in \mathscr{E}, \mathrm{d} N(t) \leq(\mu(i)-\mu(i) N(t)) \mathrm{d} t$, that is,

$$
N(t) \leq 1+(N(0)-1) e^{-\mu(i) t},
$$

where $N(0)=S(0)+I(0)+R(0)$. If $N(0) \in \Delta$, then $N(t) \leq 1$ holds until the first jump time $\tau_{1}$. When jumping into the next state, we know that the initial value $N\left(\tau_{1}\right) \leq 1$ will lead to $N(t) \leq 1$ for $t \in\left[\tau_{1}, \tau_{2}\right)$. Keep repeating the process, $N(t) \leq 1$ for all $t>0$. If $N(0)>1$, $1+(N(0)-1) e^{-\mu(i) t}$ will decrease with increase of $t$ for each $i \in \mathscr{E}$, then $N(t) \leq N(0)$. The proof is completed.

Because of this property, we assume that the initial value $S(0)+I(0)+R(0) \leq 1$ below.

Stability is one of the important research topics, which attracts a lot of attention of researchers. In this paper, we will discuss the stochastic stability of the model. In $[5,24]$, conditions for stochastic asymptotic stability in the large are given.

Lemma 2. Suppose that two functions $\mu(x) \in\left(\mathbb{R}^{3} ; \mathbb{R}^{+}\right)$with $\mu\left(P_{0}\right)=0$ and $V(x, i) \in \mathscr{C}^{2}\left(\mathbb{R}^{3} \times \mathscr{E} ; \mathbb{R}^{+}\right)$exist and satisfy

$$
\begin{array}{r}
\mathscr{L} V(x, i) \leq-\mu(x), \quad \forall(x, i) \in \mathbb{R}^{3} \times \mathscr{E}, \\
\lim _{|x| \longrightarrow \infty} \inf _{i \in \mathrm{E}} V(x, i)=\infty .
\end{array}
$$

Then, the equilibrium $P_{0}$ of the model is stochastic asymptotically stable in the large.

Lemma 3. Let $\left(S(t), I(t), R(t), \alpha_{t}\right)$ be the solution of model (2); then, it has

$$
\lim \sup _{t \rightarrow \infty} R(t) \leq \frac{\check{\gamma}}{\widehat{\mu}+\widehat{\theta}} \lim \sup _{t \rightarrow \infty} I(t),
$$

where $\check{\gamma}=\max _{i \in \mathscr{E}} r_{i}, \widehat{\mu}=\min _{i \in \mathscr{E}} \mu_{i}$, and $\widehat{\theta}$ is defined in the same way.

Proof. See reference [26] for proof.

Lemma 4. For the solution of model (2), the following formulas hold true: 


$$
\begin{aligned}
& \lim _{t \rightarrow \infty} \frac{1}{t} \int_{0}^{t} \frac{\sigma\left(\alpha_{s}\right) S(s)}{m_{1}\left(\alpha_{s}\right)+m_{1}\left(\alpha_{s}\right) S(s)+m_{2}\left(\alpha_{s}\right) I_{1}(s)} \mathrm{d} B(s)=0, \\
& \lim _{t \rightarrow \infty} \frac{1}{t} \int_{0}^{t} \frac{\sigma\left(\alpha_{s}\right) S(s) I(s)}{m_{1}\left(\alpha_{s}\right)+m_{1}\left(\alpha_{s}\right) S(s)+m_{2}\left(\alpha_{s}\right) I_{1}(s)} \mathrm{d} B(s)=0 .
\end{aligned}
$$

Proof. We only prove equation (11). Equation (12) can be testified in the same way. Let $M(t):=\int_{0}^{t}\left(\sigma\left(\alpha_{s}\right) S(s) / m_{1}\right.$ $\left.\left(\alpha_{s}\right)+m_{1}\left(\alpha_{s}\right) S(s)+m_{2}\left(\alpha_{s}\right) I_{1}(s)\right) \mathrm{d} B(s)$; then, it has

$\limsup t \rightarrow \infty \frac{\langle M, M\rangle_{t}}{t} \leq \limsup \sup _{t \rightarrow \infty} \frac{1}{t} \int_{0}^{t}\left(\frac{\check{\sigma}}{\widehat{m_{1}}}\right)^{2} \mathrm{~d} t<\infty$, a.s.

Thus, we get limsup $\rightarrow \infty \infty(M(t) / t)=0$.

\section{Stability of Disease-Free Equilibrium}

In this section, the global stochastic asymptotic stability under some conditions of disease-free equilibrium is studied by making use of Lyapunov functionals with regimeswitching.

For simplicity, let us define $f(S, I)=m_{1}+m_{2} S(t)+$ $m_{3} I(t)$ and $f(S, I, i)=m_{1}(i)+m_{2}(i) S(t)+m_{3}(i) I(t)$, then $m_{1}(i) \leq f(S, I, i) \leq m_{1}(i)+\left(m_{2}(i) \vee m_{3}(i)\right):=M(i)$. We also define functions as follows:

$$
A_{i}(x):=-\frac{\sigma^{2}(i)}{2 M^{2}(i)} x^{2}+\frac{\beta(i)}{m_{1}(i)} x-(\mu(i)+\gamma(i)+d(i)),
$$

and obviously,

$$
A_{i}(1):=\Xi_{i}=-\frac{\sigma^{2}(i)}{2 M^{2}(i)}+\frac{\beta(i)}{m_{1}(i)}-(\mu(i)+\gamma(i)+d(i)) .
$$

Theorem 1. For the initial value $\left(S(0), I(0), R(0), \alpha_{0}\right) \in$ $\Delta \times \mathscr{E}$, iffor every $i \in \mathscr{E}, \beta_{i} M^{2}(i) \geq \sigma_{i}^{2} m_{1}(i)$ and $\sum_{i=1}^{N} \pi_{i} \Xi_{i}<0$, i.e.,

$$
\Delta:=\frac{\sum_{i=1}^{N} \pi_{i}\left(\beta(i) / m_{1}(i)\right)}{\sum_{i=1}^{N} \pi_{i}\left[\mu(i)+\gamma(i)+d(i)+\left(\sigma^{2}(i) / 2 M^{2}(i)\right)\right]}<1,
$$

are satisfied, then the disease-free equilibrium is stochastically asymptotically stable in the large.

Proof. For $S(0), I(0), R(0) \in \Delta$, we define the Lyapunov functional with regime-switching as follows:

$$
V(S, I, R, i)=\frac{1}{2} \kappa_{1}(1-S)^{2}+\left(\frac{1}{\kappa_{2}}+\omega_{i}\right) I^{\kappa_{2}}+\frac{1}{2} \kappa_{3} R^{2},
$$

where $\kappa_{1}, \kappa_{2}, \kappa_{3}$, and $\omega_{i}$ are positive constants which will be specifically determined later. Using the generalized Ito formula to calculate directly, we can obtain that

$$
\begin{aligned}
\mathrm{d} V(S, I, R, i)= & \mathscr{L} V(S, I, R, i)-\frac{\kappa_{1} \sigma(i) S(1-S) I}{f(S, I, i)} \mathrm{d} B(t)+\frac{\left(1+\kappa_{2} \omega_{i}\right) \sigma(i) S I^{\kappa_{2}}}{f(S, I, i)} \mathrm{d} B(t), \\
\mathscr{L} V(S, I, R, i)= & -\kappa_{1}(1-S)\left(\mu(i)(1-S)-\frac{\beta(i) S I}{f(S, I, i)}+\theta(i) R\right) \mathrm{d} t+\frac{\kappa_{1} \sigma^{2}(i) S^{2} I^{2}}{2 f^{2}(S, I, i)} \\
& +\kappa_{2}\left(\frac{1}{\kappa_{2}}+\omega_{i}\right) I^{\kappa_{2}-1}\left[\frac{\beta(i) S I}{f(S, I, i)}-(\mu(i)+\gamma(i)+d(i)) I\right] \\
& +\kappa_{2}\left(\kappa_{2}-1\right)\left(\frac{1}{\kappa_{2}}+\omega_{i}\right) \frac{\sigma^{2}(i) S^{2} I^{\kappa_{2}}}{2 f^{2}(S, I, i)}+\kappa_{3} R[\gamma(i) I-(\mu(i)+\theta(i)) R] \\
& +I^{\kappa_{2}} \sum_{j=1}^{N} \omega_{j} q_{i j} .
\end{aligned}
$$


From Lemma 1, we know that $S(t), I(t), R(t)>0$ and $S+I+R \leq 1 ;$ thus,

$$
\begin{aligned}
\mathscr{L} V(S, I, R, i) \leq & -\kappa_{1} \mu(i)(1-S)^{2}+\frac{\kappa_{1} \beta(i) S I(1-S)}{f(S, I, i)}-\kappa_{1} \theta(i) I R+\frac{\kappa_{1} \sigma^{2}(i) S^{2} I^{2}}{2 f^{2}(S, I, i)} \\
& +\left(1+\kappa_{2} \omega_{i}\right) I^{\kappa_{2}} \frac{\beta(i) S}{f(S, I, i)}-\left(1+\kappa_{2} \omega_{i}\right)(\mu(i)+\gamma(i)+d(i)) I^{\kappa_{2}} \\
& +\left(\kappa_{2}-1\right)\left(1+\kappa_{2} \omega_{i}\right) \frac{\sigma^{2}(i) S^{2} I^{\kappa_{2}}}{2 f^{2}(S, I, i)}+\kappa_{3} \gamma(i) I R-\kappa_{3}(\mu(i)+\theta(i)) R^{2} \\
& +I^{\kappa_{2}} \sum_{j=1}^{N} \omega_{j} q_{i j} .
\end{aligned}
$$

For $\quad \kappa_{2} \leq 1, \quad$ then $\quad I^{2} \leq I \leq I^{\kappa_{2}}$. Choose $\kappa_{3} \leq \kappa_{1} \min _{i \in \mathscr{E}} \delta(i) / r(i)$; hence,

$$
\begin{aligned}
& \mathscr{L} V(S, I, R, i) \leq-\kappa_{1} \mu(i)(1-S)^{2}+\frac{\kappa_{1} \beta(i) I^{\kappa_{2}}}{m_{1}(i)}+\left(\kappa_{3} \gamma(i)-\kappa_{1} \theta(i)\right) I R \\
& +\frac{\kappa_{1} \sigma^{2}(i) S^{2} I^{\kappa_{2}}}{2 m_{1}^{2}(i)}+\left(1+\kappa_{2} \omega_{i}\right) I^{\kappa_{2}} \frac{\beta(i) S}{m_{1}(i)}-\left(1+\kappa_{2} \omega_{i}\right)(\mu(i)+\gamma(i)+d(i)) I^{\kappa_{2}} \\
& +\left(\kappa_{2}-1\right)\left(1+\kappa_{2} \omega_{i}\right) \frac{\sigma^{2}(i) S^{2} I^{\kappa_{2}}}{2 f^{2}(S, I, i)}-\kappa_{3}(\mu(i)+\theta(i)) R^{2}+I^{\kappa_{2}} \sum_{j=1}^{N} \omega_{j} q_{i j} \\
& \leq-\kappa_{1} \mu(i)(1-S)^{2}-\kappa_{3}(\mu(i)+\theta(i)) R^{2}+\left(1+\kappa_{2} \omega_{i}\right) I^{\kappa_{2}}\left[\frac{\kappa_{1} \beta(i)}{\left(1+\kappa_{2} \omega_{i}\right) m_{1}(i)}\right. \\
& \left.+\frac{\kappa_{1} \sigma^{2}(i) S^{2}}{2\left(1+\kappa_{2} \omega_{i}\right) m_{1}^{2}(i)}+A_{i}(S)+\frac{\kappa_{2} \sigma^{2}(i) S^{2}}{2 m_{1}^{2}(i)}+\frac{1}{1+\kappa_{2} \omega_{i}} \sum_{j=1}^{N} \omega_{j} q_{i j}\right] \\
& Q \omega=\sum_{i=1}^{N} \pi_{i} \Xi_{i}(1,1, \ldots, 1)^{T}-\Xi \\
& \mathscr{L} V(S, I, R, i) \leq-\kappa_{1} \mu(i)(1-S)^{2}-\kappa_{3}(\mu(i)+\theta(i)) R^{2} \\
& +\left(1+\kappa_{2} \omega_{i}\right) I^{\kappa_{2}}\left[\frac{\kappa_{1} \beta(i)}{\left(1+\kappa_{2} \omega_{i}\right) m_{1}(i)}\right. \\
& +\frac{\kappa_{1} \sigma^{2}(i)}{2\left(1+\kappa_{2} \omega_{i}\right) m_{1}^{2}(i)}+\frac{\kappa_{2} \sigma^{2}(i)}{2 m_{1}^{2}(i)} \\
& \left.+\sum_{i=1}^{N} \pi_{i} \Xi_{i}-\frac{\kappa_{2} \omega_{i}}{1+\kappa_{2} \omega_{i}} \sum_{j=1}^{N} \omega_{j} q_{i j}\right] .
\end{aligned}
$$
monotonically increasing in interval $[0,1]$; then, $A_{i}(S) \leq \Xi_{i}$ holds true. equation, that

$$
\sum_{j=1}^{N} \omega_{j} q_{i j}+\Xi_{i}=\sum_{i=1}^{N} \pi_{i} \Xi_{i}
$$

Therefore,

Let $\kappa_{2}$ be a sufficiently small constant such that $1+\kappa_{2} \omega_{i}>0$ and

$$
\frac{\kappa_{2} \sigma^{2}(i)}{2 m_{1}^{2}(i)}+\sum_{i=1}^{N} \pi_{i} \Xi_{i}-\frac{\kappa_{2} \omega_{i}}{1+\kappa_{2} \omega_{i}} \sum_{j=1}^{N} \omega_{j} q_{i j}<0 .
$$


Again, we choose a sufficiently small number $\kappa_{1}$ to make the following inequality holds true:

$$
\begin{aligned}
\kappa_{1} & \frac{2 \beta(i) m_{1}(i)+\sigma^{2}(i)}{2\left(1+\kappa_{2} \omega_{i}\right) m_{1}^{2}(i)}+\frac{\kappa_{2} \sigma^{2}(i)}{2 m_{1}^{2}(i)} \\
& +\sum_{i=1}^{N} \pi_{i} \Xi_{i}-\frac{\kappa_{2} \omega_{i}}{1+\kappa_{2} \omega_{i}} \sum_{j=1}^{N} \omega_{j} q_{i j}<0 .
\end{aligned}
$$

We can see from (32) that the coefficients are all negative constants. Hence, we arrive at the conclusion by taking advantage of Lemma 2.

\section{Asymptotic Behavior of Disease}

In this section, we shall study the asymptotic behavior of disease in model (2).

4.1. Extinction ofDisease. First of all, we study the conditions for disease extinction. With these conditions, we can take some measures to adjust the parameters in the model to make the disease go extinct in the long run.

Theorem 2. Assume that $\left(S(t), I(t), R(t), \alpha_{t}\right)$ is the solution to model (2); if one of the two conditions below holds true:

(1) If

$$
R_{0}^{S}:=\frac{\sum_{i=1}^{N} \pi_{i}\left(\beta^{2}(i) / 2 \sigma^{2}(i)\right)}{\sum_{i=1}^{N} \pi_{i}(\mu(i)+\gamma(i)+d(i))}<1
$$

(2) $\left(1 / m_{1}(i)+m_{2}(i)\right) \leq\left(\beta(i) / \sigma^{2}(i)\right)$ for all $i \in \mathscr{E}$, and

$$
R_{1}^{S}:=\frac{\sum_{i=1}^{N} \pi_{i}\left(\beta(i) /\left(m_{1}(i)+m_{2}(i)\right)\right)}{\sum_{i=1}^{N} \pi_{i}\left[\mu(i)+\gamma(i)+d(i)+\left(\sigma(i) /\left(2\left(m_{1}(i)+m_{2}(i)\right)^{2}\right)\right)\right]}<1 .
$$

Then, the disease will be extinct exponentially almost surely, i.e.,

$$
\lim _{t \longrightarrow \infty} I(t)=0, \quad \text { a.s. }
$$

Moreover,

$$
\begin{aligned}
\lim _{t \longrightarrow \infty} R(t)=0, & \text { a.s., } \\
\lim _{t \longrightarrow \infty} S(t)=1, & \text { a.s. }
\end{aligned}
$$

Proof. For case (1), applying the Ito formula to function $\ln I(t)$, we can obtain that

$$
\begin{aligned}
\mathrm{d} \ln I(t)= & \mathscr{L} \ln I(t) \mathrm{d} t+\frac{\sigma\left(\alpha_{t}\right) S(t)}{f\left(S, I, \alpha_{t}\right)} \mathrm{d} B(t), \\
\mathscr{L} \ln I(t)= & \frac{\beta\left(\alpha_{t}\right) S(t)}{f\left(S, I, \alpha_{t}\right)}-\left[\mu\left(\alpha_{t}\right)+\gamma\left(\alpha_{t}\right)+d\left(\alpha_{t}\right)\right] \\
& -\frac{\sigma^{2}\left(\alpha_{t}\right) S^{2}}{2 f^{2}\left(S, I, \alpha_{t}\right)} .
\end{aligned}
$$

Define

$$
g\left(S, I, \alpha_{t}\right)=S / f\left(S, I, \alpha_{t}\right)
$$

then $0<g\left(S, I, \alpha_{t}\right) \leq\left(1 / m_{1}\left(\alpha_{t}\right)+m_{2}\left(\alpha_{t}\right)\right)$ and

$$
\begin{aligned}
\mathscr{L} \ln I(t)= & -\frac{\sigma^{2}\left(\alpha_{t}\right)}{2}\left[g\left(S, I, \alpha_{t}\right)-\frac{\beta\left(\alpha_{t}\right)}{\sigma^{2}\left(\alpha_{t}\right)}\right]^{2}+\frac{\beta^{2}\left(\alpha_{t}\right)}{2 \sigma^{2}\left(\alpha_{t}\right)} \\
& -\left[\mu\left(\alpha_{t}\right)+\gamma\left(\alpha_{t}\right)+d\left(\alpha_{t}\right)\right] \\
\leq & \frac{\beta^{2}\left(\alpha_{t}\right)}{2 \sigma^{2}\left(\alpha_{t}\right)}-\left[\mu\left(\alpha_{t}\right)+\gamma\left(\alpha_{t}\right)+d\left(\alpha_{t}\right)\right] .
\end{aligned}
$$

Integrating for the both sides of (30) from 0 to $t$ followed by dividing both sides by $t$ yields

$$
\begin{aligned}
\frac{\ln I(t)}{t} \leq & \frac{\ln I(0)}{t}+\frac{1}{t} \int_{0}^{t}\left[\frac{\beta^{2}\left(\alpha_{s}\right)}{2 \sigma^{2}\left(\alpha_{t}\right)}-\left[\mu\left(\alpha_{s}\right)+\gamma\left(\alpha_{s}\right)+d\left(\alpha_{s}\right)\right]\right] \mathrm{d} s \\
& +\frac{1}{t} \int_{0}^{t} \frac{\sigma\left(\alpha_{s}\right) S(s)}{f\left(S, I, \alpha_{s}\right)} \mathrm{d} B((s)) .
\end{aligned}
$$

From ergodic properties of Markov chains $\left\{\alpha_{t}\right\}$ and Lemma 4 , we can obtain

$$
\limsup _{t \rightarrow \infty} \frac{\ln I(t)}{t} \leq \sum_{i=1}^{N} \pi_{i}\left[\frac{\beta^{2}(i)}{2 \sigma^{2}(i)}-[\mu(i)+\gamma(i)+d(i)]\right] .
$$

With the help of (26), we get that $\lim _{t \rightarrow \infty}(\ln I(t) / t)<0$, a.s., which means the disease will go to extinction exponentially almost surely.

For case (2), we can obtain from (31) that if $\left(1 / m_{1}(i)+\right.$ $\left.m_{2}(i)\right) \leq\left(\beta(i) / \sigma^{2}(i)\right)$ for all $i \in \mathscr{E}$, then

$$
\begin{aligned}
\mathscr{L} \ln I(t) \leq & \frac{\beta\left(\alpha_{t}\right)}{m_{1}\left(\alpha_{t}\right)+m_{2}\left(\alpha_{t}\right)}-\left[\mu\left(\alpha_{t}\right)+\gamma\left(\alpha_{t}\right)+d\left(\alpha_{t}\right)\right] \\
& -\frac{\sigma^{2}\left(\alpha_{t}\right)}{2\left(m_{1}\left(\alpha_{t}\right)+m_{2}\left(\alpha_{t}\right)\right)^{2}} .
\end{aligned}
$$

Thus, 


$$
\begin{aligned}
\frac{\ln I(t)}{t} \leq & \frac{\ln I(0)}{t}+\frac{1}{t} \int_{0}^{t}\left[\frac{\beta\left(\alpha_{s}\right)}{m_{1}\left(\alpha_{s}\right)+m_{2}\left(\alpha_{s}\right)}-\left[\mu\left(\alpha_{s}\right)+\gamma\left(\alpha_{s}\right)\right.\right. \\
& \left.\left.+d\left(\alpha_{s}\right)\right]\right] \mathrm{d} s \\
& -\frac{1}{t} \int_{0}^{t}\left[\frac{\sigma^{2}\left(\alpha_{s}\right)}{2\left(m_{1}\left(\alpha_{s}\right)+m_{2}\left(\alpha_{s}\right)\right)^{2}}\right] \mathrm{d} s \\
& +\frac{1}{t} \int_{0}^{t} \frac{\sigma\left(\alpha_{s}\right) S(s)}{f\left(S, I, \alpha_{s}\right)} \mathrm{d} B(s) .
\end{aligned}
$$

Therefore, conclusion (28) can be obtained by taking advantage of (27).

The equality $\lim _{t \rightarrow \infty} R(t)=0$, a.s. Followed by utilizing Lemma 3, next we prove the second conclusion of (29).

Assume that $A=\left\{\omega \in \Omega: \lim _{t \rightarrow \infty} I(t)=0\right.$, $\left.\lim _{t \rightarrow \infty} R(t)=0\right\}$; then, the above conclusions tell us that $\mathbb{P}(A)=1$. Thus, for any constant $\epsilon>0$ and $\omega \in A$, there exists a positive constant $T=T(\omega, \varepsilon)$ such that

$$
I(t, \omega) \leq \varepsilon, R(t, \omega) \leq \varepsilon, \quad \forall t \geq T .
$$

Therefore, according to (7), for $t \geq T$, one has

$$
\begin{aligned}
\frac{\mathrm{d} N(t)}{\mathrm{d} t}= & \mu\left(\alpha_{t}\right)-\mu\left(\alpha_{t}\right) N(t)-d\left(\alpha_{t}\right) I(t) \geq \mu\left(\alpha_{t}\right) \\
& -\mu\left(\alpha_{t}\right) N(t)-\check{d} \varepsilon .
\end{aligned}
$$

No matter how large the $t$ is, (38) belongs to one of the following formulas:

$$
\frac{\mathrm{d} N(t)}{\mathrm{d} t} \geq \mu(i)-\mu(i) N(t)-\check{d} \varepsilon, \quad i \in \mathrm{E} .
$$

Applying the variation of constants formula to (39) yields

$$
N(t) \geq \frac{\mu(i)-\check{d} \varepsilon}{\mu(i)}+\left(N(T)-\frac{\mu(i)-\check{d} \varepsilon}{\mu(i)}\right) \exp \{-\mu(i)(t-T)\} .
$$

For any $i \in \mathscr{E}$ and the arbitrariness of $\varepsilon$, the right side of (40) tends to 1 when $t$ goes to infinity; then, it has

$$
\liminf _{t \rightarrow \infty} N(t) 1, \quad \forall \omega \in A .
$$

which implies that

$$
\liminf _{t \rightarrow \infty} N(t) \geq 1, \quad \text { a.s. }
$$

Recalling the assertion $N(t) \leq 1$ in Lemma 1 and the fact that $N(t)=S(t)+I(t)+R(t)$, the conclusion that $\lim _{t \rightarrow \infty} S(t)=1$, a.s. can be obtained.

\section{Remark 1.}

(1) From the first condition above in Theorem 2, we can see that if the intensity of stochastic perturbation is sufficiently large, $R_{0}^{S}<1$ is sure to work; then, the disease will be extinct, which shows that the stochastic perturbation has an important influence on the model. When the intensity of stochastic perturbation is small, the disease will still be extinct if the second condition is satisfied.

(2) We can see from the expressions of (16) and (27) that $R_{1}^{S} \leq \Delta$; thus, if $\Delta<1$, then $R_{1}^{S}<1$, which means that stochastically asymptotically stability in the large will also make the disease go extinct.

4.2. Permanence of Disease. Next, we move forward to analyze the conditions of disease persistence. First of all, a definition about persistence is given.

Definition 1. The disease in model (2) is called persistent in the mean if there exists a constant $\eta>0$ such that

$$
\liminf _{t \rightarrow \infty} \frac{1}{t} \int_{0}^{t} I(u) d u \geq \eta, \quad \text { a.s. }
$$

Theorem 3. Assume that $\left(S(t), I(t), R(t), \alpha_{t}\right)$ is the solution to model (2) with initial value $\left(S(0), I(0), R(0), \alpha_{0}\right)$ and $0<S(0)+I(0)+R(0) \leq 1$. If

$$
R_{2}^{S}:=\frac{\sum_{i=1}^{N} \pi_{i} \mu(i)}{\lambda \sum_{i=1}^{N} \pi_{i}\left[\mu(i)+d(i)+r(i)+\left(\sigma^{2}(i) / 2\left(m_{1}(i)+m_{2}(i)\right)^{2}\right)\right]}>1,
$$

where $\lambda=\max _{i \in \mathscr{E}}\left\{\mu(i)\left(m_{1}(i)+m_{2}(i)\right) / \beta(i)\right\}$, then the disease will be persistent in the mean.

Proof. We prove this theorem in two steps. First, let us prove that the following inequality holds true for a certain positive constant $C$ :

$$
H(S, I, i):=\left[(\lambda-I) \frac{\beta(i)}{m_{1}(i)+m_{2}(i) S+m_{3}(i) I}-\mu(i)\right] S+C I \geq 0 .
$$

where $I, S \in[0,1], i \in \mathscr{E}$.

It is clear that $H(S, 0, i)=\left[\left(\lambda \beta(i) / m_{1}(i)+m_{2}(i) S\right)-\mu\right.$ (i) $] S \geq\left[\left(\lambda \beta(i) / m_{1}(i)+m_{2}(i)\right)-\mu(i)\right] S \geq 0$, and

$$
\begin{aligned}
\frac{\partial H}{\partial I} & =\left[-\frac{\beta(i) S\left[m_{1}(i)+m_{2}(i) S+\lambda m_{3}(i)\right]}{\left(m_{1}(i)+m_{2}(i) S+m_{3}(i) I\right)^{2}}\right]+C \\
& \geq C-\max _{i \in \mathrm{E}}\left[\frac{\beta(i)\left[m_{1}(i)+m_{2}(i)+\lambda m_{3}(i)\right]}{m_{1}^{2}(i)}\right] .
\end{aligned}
$$

We can see easily that, for sufficiently large constant $C$, $\partial H / \partial I>0$; then, from the monotone increasing property of $H(S, I, i)$, there exists a constant $\epsilon, H(S, I, i)>0$ for $S \in[0,1], I \in[0, \varepsilon]$, and all $i \in \mathscr{E}$. Next, we prove that $H(S, I, i)>0$ for $S \in[0,1], \quad I \in[\mathcal{E}, 1]$, and all $i \in \mathscr{E}$. According to the expression of $H(S, I, i)$, 


$$
\begin{array}{r}
H(S, I, i) \geq-\frac{\beta(i) S I}{m_{1}(i)+m_{2}(i) S+m_{3}(i) I}-\mu(i) S+C I \\
\geq C \epsilon-\max _{i \in \mathscr{E}}\left[\frac{\beta(i)}{m_{1}(i)}+\mu(i)\right] .
\end{array}
$$

For sufficiently large constant $C, H(S, I, i)>0$ for $I \in[\varepsilon, 1]$. Now, inequality (45) has been proved.

In what follows, we will demonstrate our conclusion in the theorem. From the first equation of model (2), we know that

$$
\begin{aligned}
\mathrm{d} S(t) \geq & \left(\mu\left(\alpha_{t}\right)-\mu\left(\alpha_{t}\right) S(t)-\frac{\beta\left(\alpha_{t}\right) S(t) I(t)}{f\left(S, I, \alpha_{t}\right)}\right) \mathrm{d} t \\
& -\frac{\sigma\left(\alpha_{t}\right) S(t) I(t)}{f\left(S, I, \alpha_{t}\right)} \mathrm{d} B(t) \\
\geq & \left(\mu\left(\alpha_{t}\right)-\frac{\lambda \beta\left(\alpha_{t}\right) S(t)}{f\left(S, I, \alpha_{t}\right)}-C I(t)\right) \mathrm{d} t \\
& -\frac{\sigma\left(\alpha_{t}\right) S(t) I(t)}{f\left(S, I, \alpha_{t}\right)} \mathrm{d} B(t) .
\end{aligned}
$$

According to the result of (31), we obtain that

$$
\begin{aligned}
\mathscr{L}(\lambda \ln I(t)) \mathrm{d} t= & \frac{\lambda \beta\left(\alpha_{t}\right) S(t)}{f\left(S, I, \alpha_{t}\right)}-\lambda\left[\mu\left(\alpha_{t}\right)+\gamma\left(\alpha_{t}\right)+d\left(\alpha_{t}\right)\right] \\
& -\frac{\lambda \sigma^{2}\left(\alpha_{t}\right) S^{2}}{2 f^{2}\left(S, I, \alpha_{t}\right)} \\
\geq & \left(\mu\left(\alpha_{t}\right)-\lambda\left[\mu\left(\alpha_{t}\right)+\gamma\left(\alpha_{t}\right)+d\left(\alpha_{t}\right)\right.\right. \\
& +\frac{\sigma^{2}\left(\alpha_{t}\right)}{\left.\left.2\left(m_{1}\left(\alpha_{t}\right)+m_{2}\left(\alpha_{t}\right)\right)^{2}\right]\right) \mathrm{d} t} \\
& -C I(t) \mathrm{d} t-\mathrm{d} S(t)-\frac{\sigma\left(\alpha_{t}\right) S(t) I(t)}{f\left(S, I, \alpha_{t}\right)} \mathrm{d} B(t) .
\end{aligned}
$$

Making use of the result of (49), integrating for the both sides of $\mathrm{d}(\lambda \ln I(t))=\mathscr{L}(\lambda \ln I(t)) \mathrm{d} t+\lambda\left(\sigma\left(\alpha_{t}\right) S(t) I(t) /\right.$ $\left.f\left(S, I, \alpha_{t}\right)\right) \mathrm{d} B(t)$ from 0 to $t$ and dividing by $t$, one has

$$
\begin{aligned}
C \frac{1}{t} \int_{0}^{t} I(s) \mathrm{d} s \geq & \frac{1}{t} \int_{0}^{t}\left(\mu\left(\alpha_{s}\right)-\lambda\left[\mu\left(\alpha_{s}\right)+\gamma\left(\alpha_{s}\right)+d\left(\alpha_{s}\right)\right]\right) \mathrm{d} s \\
& -\frac{1}{t} \int_{0}^{t} \frac{\lambda \sigma^{2}\left(\alpha_{s}\right)}{2\left(m_{1}\left(\alpha_{s}\right)+m_{2}\left(\alpha_{s}\right)\right)^{2}} \\
& -\frac{\lambda(\ln I(t)-\ln I(0))}{t} \\
& -\frac{S(t)-S(0)}{t}+\frac{1}{t} \int_{0}^{t} \frac{(\lambda-1) \sigma\left(\alpha_{s}\right) S(s) I(s)}{f\left(S, I, \alpha_{s}\right)} \mathrm{d} B(s) .
\end{aligned}
$$

Using the boundedness of $S(t), I(t)$, and Lemma 4 along with the ergodic property of Markov chain $\left(\alpha_{t}\right)$, we take the limit inferior for both sides; then,

$$
\begin{aligned}
\liminf _{t \rightarrow \infty} \frac{1}{t} \int_{0}^{t} I(s) \mathrm{d} s \geq & \frac{1}{C} \sum_{i=1}^{N} \pi_{i}[\mu(i)-\lambda(\mu(i)+\gamma(i)+d(i) \\
& \left.\left.+\frac{\sigma^{2}(i)}{2\left(m_{1}(i)+m_{2}(i)\right)^{2}}\right)\right]
\end{aligned}
$$

Therefore, if (44) is satisfied, then $\liminf _{t \rightarrow \infty}(1 / t) \int_{0}^{t} I(s) \mathrm{d} s>0$, which means the disease will be persistent in the mean.

The proof is completed.

Corollary 1. From the expressions of $R_{1}^{S}$ in (27) and $R_{2}^{S}$ in (44), we have $R_{1}^{S} \geq R_{2}^{S} . R_{1}^{S}=R_{2}^{S}$ holds if and only if $\mu(i), \beta(i)$, $m_{1}(i)$, and $m_{2}(i)$ in the formulas are fixed constants.

Proof

$$
\begin{aligned}
\frac{R_{1}^{S}}{R_{2}^{S}} & =\frac{\lambda \sum_{i=1}^{N} \pi_{i}\left(\beta(i) / m_{1}(i)+m_{2}(i)\right)}{\sum_{i=1}^{N} \pi_{i} \mu(i)} \\
& =\frac{\max \left\{\left(\mu(i)\left(m_{1}(i)+m_{2}(i)\right) / \beta(i)\right)\right\} \sum_{i=1}^{N}\left(\beta(i) / \mu(i)\left(m_{1}(i)+m_{2}(i)\right)\right) \pi_{i} \mu(i)}{\sum_{i=1}^{N} \pi_{i} \mu(i)} \\
& \geq \frac{\sum_{i=1}^{N} \pi_{i} \mu(i)}{\sum_{i=1}^{N} \pi_{i} \mu(i)}=1 .
\end{aligned}
$$




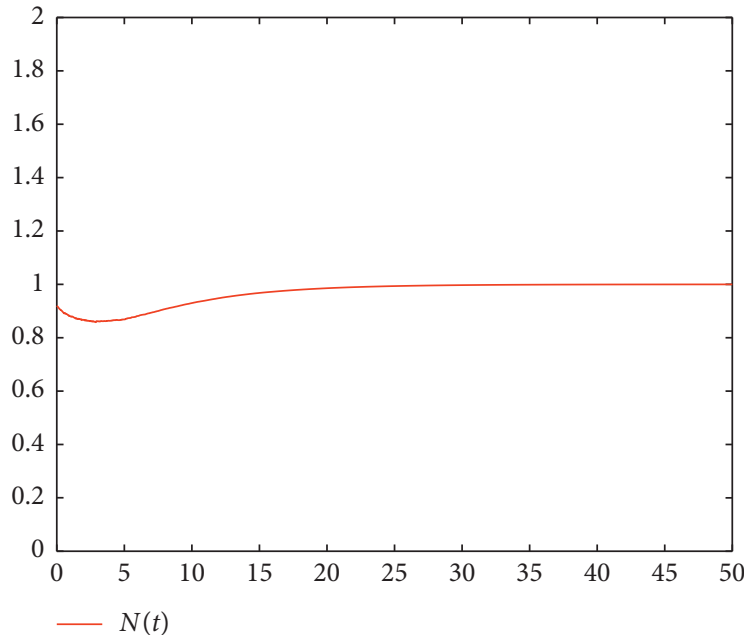

(a)

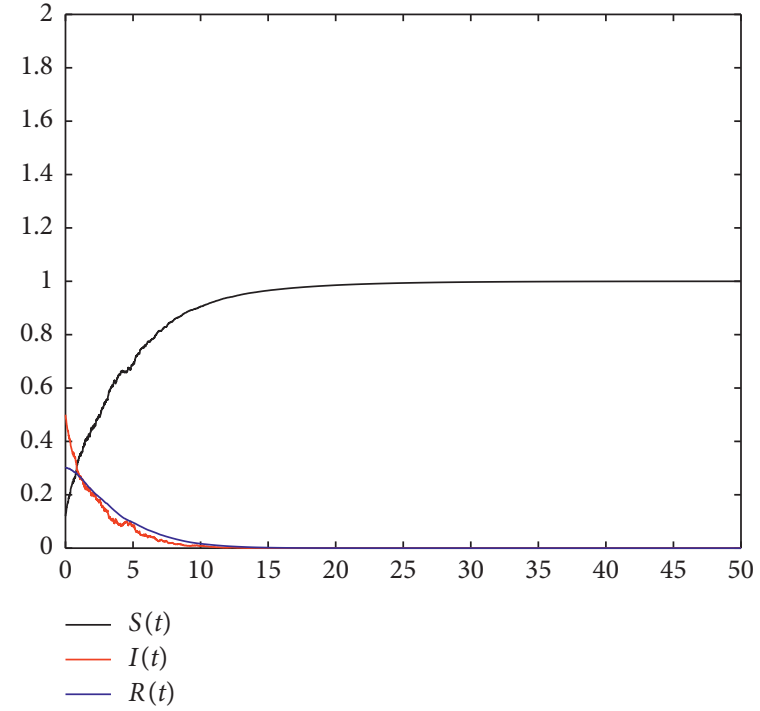

(b)

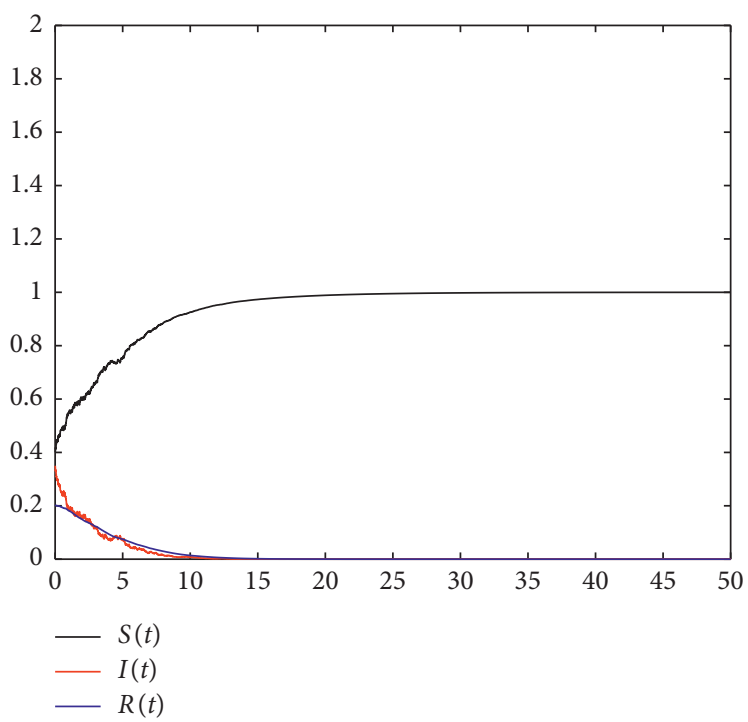

(c)

Figure 1: The trajectories with parameters in Example 1: (a) the trajectory of $N(t)$; (b and c) the trajectories with different initial values.

As can be seen from the above proof, if and only if $\max _{i \in \mathscr{E}}\left(\mu(i)\left(m_{1}(i)+m_{2}(i)\right) / \beta(i)\right)=\left(\mu(i) m_{1}(i)+m_{2}(i) / \beta(i)\right)$ for every $i \in \mathscr{E}, R_{1}^{S}=R_{2}^{S}$ holds true.

\section{Remark 2.}

(1) $R_{1}^{S} \geq R_{2}^{S}$ means that Theorems 2 and 3 are not in conflict. If $R_{1}^{S}<1$, then $R_{2}^{S}<1$, and the disease will die out. If $R_{2}^{S}>1$, then $R_{1}^{S}>1$, and the disease will be persistent.

(2) If there is no regime-switching in model (2), i.e., there is only one environment, then

$$
R_{1}^{S}=R_{2}^{S}:=R=\left(\frac{\left(\beta /\left(m_{1}+m_{2}\right)\right)}{\mu+\gamma+d+\left(\sigma^{2} / 2\left(m_{1}+m_{2}\right)^{2}\right)}\right) \text {, }
$$

can be considered as the threshold of disease persistence and extinction in the model. We can see from formula
(53) that $R$ will increase with the increase of contact coefficient $\beta$. When $R>1$, the disease will be persistent in the long run. Therefore, it will be one of the important ways to control infectious diseases to reduce the value of transmission coefficient $\beta$ by isolating the infected and limiting the people's going out, which is widely used when SARS virus and new coronavirus pneumonia spread in China.

Due to the existence of regime-switching, the behavior of disease will have different phenomena. Examples 2 and 3 will reveal some interesting things.

\section{Examples and Simulations}

In this section, some examples will be proposed and their numerical simulations are presented to verify our theoretical results above. 


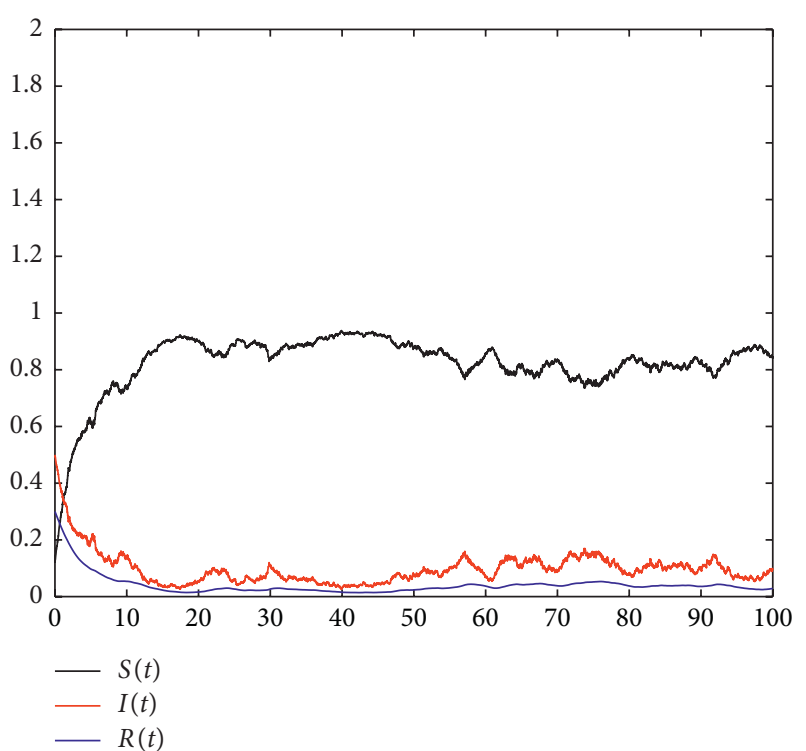

(a)

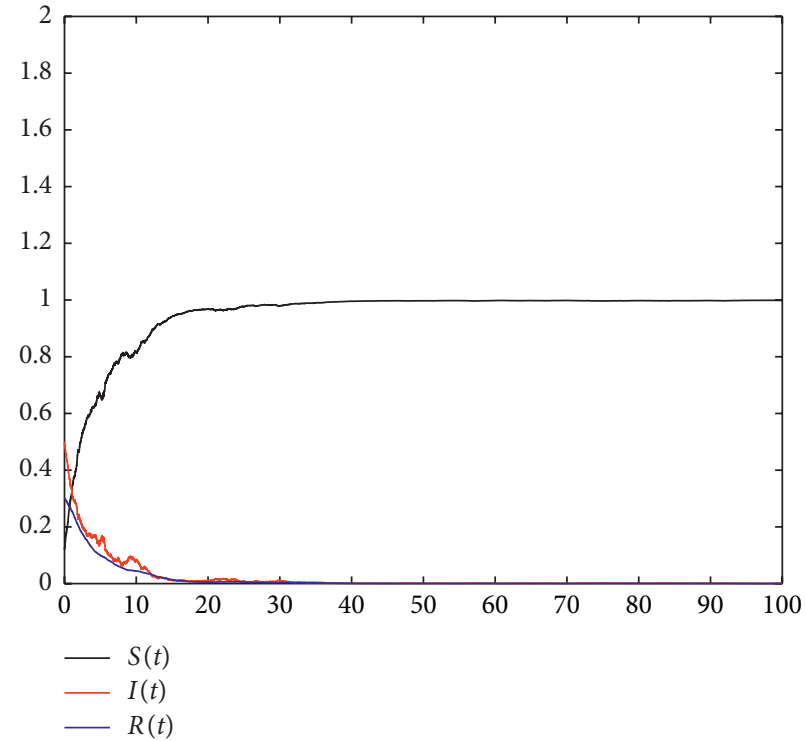

(b)

FIGURE 2: (a) The trajectory of Environment 1 with $R=1.3483>1$ and all parameters in Example 2; (b) the trajectory of the regimeswitching model with $R_{1}^{S}<1$ with parameters in Example 2.

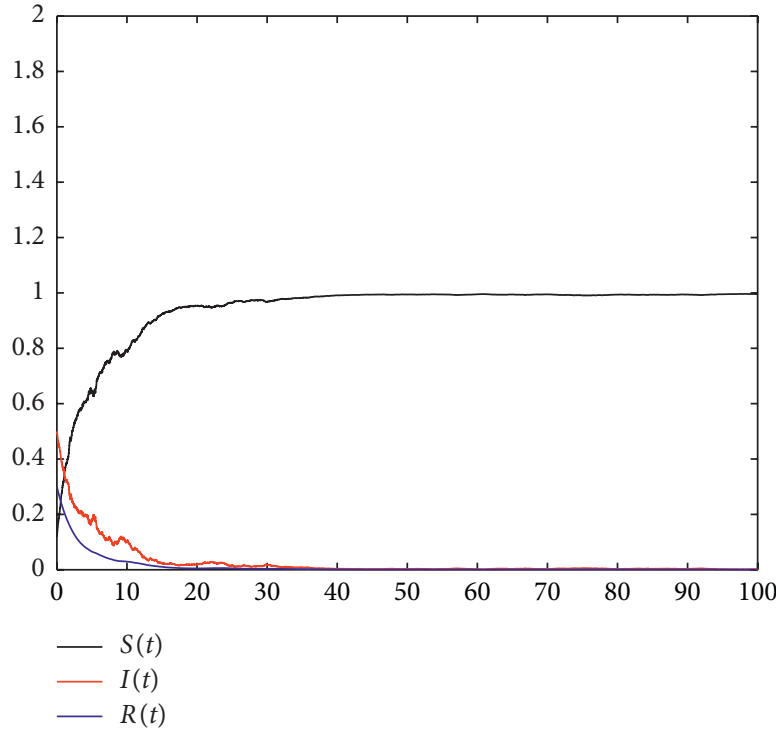

(a)

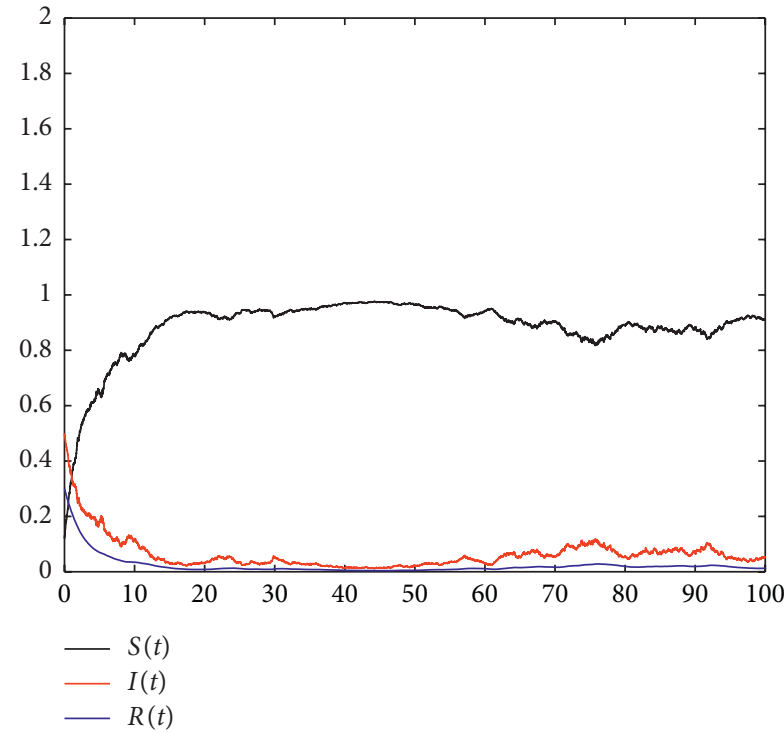

(b)

Figure 3: (a) The trajectory of Environment 1 with $R=0.9756<1$ and parameters in Example 3; (b) the trajectory of the regime-switching model with $R_{1}^{S}>1$ and parameters in Example 3.

Let $\left(\alpha_{t}\right)_{t \geq 0}$ be a Markov chain taking values in the space $\mathscr{E}=\{1,2\}$ with the transition rate matrix:

$$
Q=\left(\begin{array}{cc}
-m & m \\
n & -n
\end{array}\right) \text {. }
$$

Hence, there exits two environments, say, Environment 1 and Environment 2, and its stationary distribution $\pi=\left(\pi_{1}, \pi_{2}\right)=((n / m+n),(m / m+n))$.

Example 1. First, we verify the results of Lemma 1 and Theorem 1. Assume that $m=2$ and $n=1$; then, the stationary distribution $\pi=\left(\pi_{1}, \pi_{2}\right)=(1 / 2,2 / 3)$. Let $S(0)=0.12, I(0)=0.5, R(0)=0.3, \mu(1)=0.15, \mu(2)=0.2$, $\beta(1)=0.5, \beta(2)=0.3, d(1)=0.15, d(2)=0.1, \theta(1)=0.4$, $\theta(2)=0.25, \gamma(1)=0.3, \gamma(2)=0.4, \sigma(1)=0.8, \sigma(1)=1$, $m_{1}(1)=1, m_{1}(2)=1, m_{2}(1)=1, m_{2}(2)=2, m_{3}(1)=2$, and $\quad m_{3}(2)=3$; $\quad$ thus, $\quad S(0)+I(0)+R(0)<1$, $\beta_{i} M^{2}(i) \geq \sigma_{i}^{2} m(i)$, and $\sum_{i=1}^{N} \pi_{i} \Xi_{i}<0$ are satisfied for $i=1,2$. According to Lemma $1, N(t)=S(t)+I(t)+R(t) \leq 1$ will always hold, see Figure 1(a). In order to verify the stability of the model, we select two different sets of parameters. The parameters in set 1 are as above, and its 
trajectory is shown Figure 1(b). Parameters in the other set are the same except $\beta(1)=0.5, \beta(2)=0.3, m=1$, and $n=2$. Moreover, the initial values are different; let $S(0)=0.4$, $I(0)=0.35$, and $R(0)=0.2$, and its trajectory can be seen in Figure 1(c).

Example 2. Next, let us test Theorem 2. Since the parameters of the first conclusion can be selected from Example 1, we only verify the second conclusion. Assume that $m=1$ and $n=2$, and let $\beta(1)=0.6, \beta(2)=0.4, \gamma(1)=0.2, \gamma(2)=0.4$, $\sigma(1)=0.3, \sigma(1)=5, m_{2}(1)=0, m_{2}(2)=2, m_{3}(1)=2$, and $m_{3}(2)=3$; the other parameters are the same as those in Example $\quad 1$. Then, $\quad R_{1}^{S}=0.8313<1 \quad$ and $\left(1 / m_{1}(i)+m_{2}(i)\right) \leq\left(\beta(i) / \sigma^{2}(i)\right)$ are satisfied, although $R=$ $1.3483>1$ in Environment 1. The data means that the disease will be persistent in Environment 1 (see Figure 2(a)), but it will go to extinction as a result of regime-switching, which shows the important role of Markovian Switching. The simulation can be seen in Figure 2(b).

Example 3. Finally, we verify Theorem 3. Let $m=3, n=12$, $\mu(1)=0.2, \mu(2)=0.3, \beta(1)=0.9, \beta(2)=0.95, d(1)=0.1$, $d(2)=0.08, \gamma(1)=0.15, \gamma(2)=0.2, \sigma(1)=0.3, \sigma(2)=0.2$, $m_{1}(1)=1, m_{1}(2)=1, m_{2}(1)=1, m_{2}(2)=0, m_{3}(1)=2$, and $m_{3}(2)=1$. By calculation, we can get $R_{2}^{S}=1.0123>1$ while $R=0.9756<1$ in Environment 1, which means the disease will die out in Environment 1 (see Figure 3(a)), but it will continue as a result of Markovian switching, see Figure 3(b).

\section{Conclusions}

In this paper, we study the regime-switching SIRS model with the Beddington-DeAngelis incidence rate. We first prove that the model we discuss has a unique positive solution. Secondly, we give the conditions of global stochastic asymptotic stability by the Lyapunov method. Then, the thresholds of disease behavior are given by some useful inequality technique. Finally, some examples are given and numerical simulations are presented to confirm our conclusions.

In addition, some more topics are worth further studying. More complex models can be considered to better reflect the actual situation, for example, the model with more general incidence rate, more perturbations such as jump noise, or the model with the effect of time delay. We will keep these for our future research.

\section{Data Availability}

The data used to support the findings of this study are included within the article.

\section{Conflicts of Interest}

The authors declare that they have no conflicts of interest.

\section{Acknowledgments}

This work was supported by the Science and Technology projects of Jiangxi Provincial Education Department (nos. GJJ181105 and GJJ191145) and National Natural Science Foundation of China (no. 11661065).

\section{References}

[1] B. Buonomo and S. Rionero, "On the Lyapunov stability for SIRS epidemic models with general nonlinear incidence rate," Applied Mathematics and Computation, vol. 217, no. 8, pp. 4010-4016, 2010.

[2] J. Q. Li, Y. L. Yang, Y. N. Xiao, and S. Liu, "A class of Lyapunov functions and the global stability of some epidemic models with nonlinear incidence," Journal of Applied Analysis and Computation, vol. 6, no. 1, pp. 38-46, 2016.

[3] Q. Tang, Z. Teng, and X. Abdurahman, "A new Lyapunov function for SIRS epidemic models," Bulletin of the Malaysian Mathematical Sciences Society, vol. 40, no. 1, pp. 237-258, 2017.

[4] X. R. Mao, Stochastic Differential Equation and Applications, Horwood, Chichester, England, 1997.

[5] X. R. Mao and C. G. Yuan, Stochastic Differential Equations with Markovian Switching, Imperial College Press, London, UK, 2006

[6] Y. Cai, Y. Kang, M. Banerjee, and W. Wang, "A stochastic SIRS epidemic model with infectious force under intervention strategies," Journal of Differential Equations, vol. 259, no. 12, pp. 7463-7502, 2015.

[7] A. Lahrouz and A. Settati, "Necessary and sufficient condition for extinction and persistence of SIRS system with random perturbation," Applied Mathematics and Computation, vol. 233, pp. 10-19, 2014.

[8] Q. Liu, "The threshold of a stochastic Susceptible-Infective epidemic model under regime switching," Nonlinear Analysis: Hybrid Systems, vol. 21, pp. 49-58, 2016.

[9] Q. Liu, D. Jiang, and N. Shi, "Threshold behavior in a stochastic SIQR epidemic model with standard incidence and regime switching," Applied Mathematics and Computation, vol. 316, pp. 310-325, 2018.

[10] Q. Liu, D. Jiang, T. Hayat, and A. Alsaedi, "Dynamical behavior of a hybrid switching SIS epidemic model with vaccination and Lévy jumps," Stochastic Analysis and Applications, vol. 37, no. 3, pp. 388-411, 2019.

[11] X. Guo and J. Luo, "Stationary distribution and extinction of sir model with nonlinear incident rate under markovian switching," Physica A: Statistical Mechanics and Its Applications, vol. 505, pp. 471-481, 2018.

[12] D. Li, S. Liu, and J. A. Cui, "Threshold dynamics and ergodicity of an SIRS epidemic model with markovian switching," Journal of Differential Equations, vol. 263, no. 12, pp. 88738915, 2017.

[13] Y. Lin, D. Jiang, and M. Jin, "Stationary distribution of a stochastic SIR model with saturated incidence and its asymptotic stability," Acta Mathematica Scientia, vol. 35, no. 3, pp. 619-629, 2015.

[14] H. K. Qi, X. Z. Meng, and Z. B. Chang, "Markov semigroup approach to the analysis of a nonlinear stochastic plant disease model," Electronic Journal of Differential Equations, vol. 2019, no. 116, pp. 1-19, 2019.

[15] X. Mao, G. Marion, and E. Renshaw, "Environmental Brownian noise suppresses explosions in population 
dynamics," Stochastic Processes and Their Applications, vol. 97, no. 1, pp. 95-110, 2002.

[16] Y. Cai, Y. Kang, and W. M. Wang, "A stochastic SIRS epidemic model with nonlinear incidence rate," Applied Mathematics and Computation, vol. 305, pp. 221-240, 2017.

[17] N. H. Du and N. N. Nhu, "Permanence and extinction of certain stochastic SIR models perturbed by a complex type of noises," Applied Mathematics Letters, vol. 64, pp. 223-230, 2017.

[18] J. H. Bao and J. H. Shao, "Permanence and extinction of regime-switching predator-prey models," SIAM Journal on Mathematical Analysis, vol. 48, no. 1, pp. 725-739, 2017.

[19] A. Lahrouz, A. Settati, and P. S. Mandal, "Dynamics of a switching diffusion modified Leslie-Gower predator-prey system with Beddington-DeAngelis functional response," Nonlinear Dynamics, vol. 85, no. 2, pp. 853-870, 2016.

[20] Z. Chang, X. Meng, and X. Lu, "Analysis of a novel stochastic SIRS epidemic model with two different saturated incidence rates," Physica A: Statistical Mechanics and Its Applications, vol. 472, pp. 103-116, 2017.

[21] A. Lahrouz and A. Settati, "Asymptotic properties of switching diffusion epidemic model with varying population size," Applied Mathematics and Computation, vol. 219, no. 24, pp. 11134-11148, 2013.

[22] T. Hou and J. Shao, "Heavy tail and light tail of Cox-IngersollRoss processes with regime-switching," Science China Mathematics, vol. 63, no. 6, pp. 1169-1180, 2019.

[23] X. Li and G. Yin, "Logistic models with regime switching: permanence and ergodicity," Journal of Mathematical Analysis and Applications, vol. 441, no. 2, pp. 593-611, 2016.

[24] R. Z. Khasminskii, C. Zhu, and G. Yin, "Stability of regimeswitching diffusions," Stochastic Processes and Their Applications, vol. 117, no. 8, pp. 1037-1051, 2007.

[25] G. Yin and C. Zhu, Hybrid Switching Diffusions: Properties and Applications, Springer, New York, NY, USA, 2010.

[26] F. Wang and Z. M. Liu, "Dynamical behavior of stochastic SIRS model with two different incidence rates and Markovian switching," Advances in Difference Equations, vol. 2019, no. 1, 322 pages, 2019. 